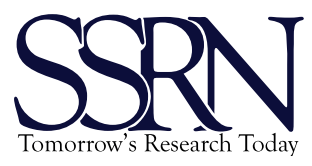

11th Conference on Learning Factories, CLF2021

\title{
Enabling distance learning and remote collaboration in layout and process planning
}

\author{
Zsolt Kemény a,*, Richárd Beregi ${ }^{a}$, Mátyás Hajós ${ }^{\mathrm{a}}$, János Csempesz ${ }^{\mathrm{a}}$, János Nacsa ${ }^{\mathrm{a}}$ \\ ${ }^{a}$ Centre of Excellence in Production Informatics and Control, Institute for Computer Science and Control (SZTAKI), \\ Kende u. 13-17., 1111 Budapest, Hungary
}

\begin{abstract}
Focusing on layout and process planning in collaborative environments, the development of infrastructure and didactic content for hands-on courses has been in progress for years at SZTAKI, with the inclusion of two sites in Budapest and Győr, respectively. Since 2019, a summer school program has been in preparation involving the Pilot Factory in Vienna, maintained by Fraunhofer Austria and TU Wien. While the pandemic has forced on-site education to be postponed, it also raised a lasting need for enabling distance learning and remote collaboration for a learning factory infrastructure which has hitherto been developed for on-site, hands-on activities as the primary consideration. The paper presents extensions addressing these needs in three different dimensions: tailoring task complexity by adequate shaping of free decision spaces, improving transferability of the problems to virtual or off-site solution platforms, and reorganization of work and cross-site collaboration. The concepts are illustrated by the example of a summer school course in layout and process planning for collaborative assembly stations, planned to take place in July/August 2021.
\end{abstract}

(C) 2021 The Authors. This is an open access article.

Peer-review statement: Peer-review under responsibility of the scientific committee of the 11th Conference on Learning Factories 2021.

Keywords: Layout planning; process planning; course development; distance learning; remote collaboration

\section{Introduction}

The impact of the COVID-19 pandemic has taken a heavy toll on higher education, to a major part due to on-site attendance being restricted or made entirely impossible by lockdowns and travel restrictions. As a comprehensive IAU survey [1] revealed, various virtual mobility or online education solutions have been widely implemented as an immediate remedy. While this has sufficiently addressed problems - or even opened up new opportunities-in many domains, other branches were still left with major challenges deeply affecting core sections of their educational profile. Notably, these are areas where personal attendance and hands-on experience with site-bound, physical equipment are crucial in attaining key skills and knowledge of the domain. Many of today's learning factories are, therefore, in a vulnerable position, as they rely substantially on hands-on activities, often with physical equipment that carries out

\footnotetext{
* Corresponding author. Tel.: +36-1-279-6180.

E-mail address: kemeny@sztaki.hu
} 
real production processes, and is site-bound as a consequence. Over decades of gradual development, these facilities have successfully responded to existing demands $[2,3,4]$ —in particular, for practical skills and attitudes which have been less effectively addressed by conventional higher education or training [5].

Prior to the pandemic, limitations of personal attendance have not presented a profound issue, and the site-bound character of facilities rarely meant more than a logistics bottleneck that could be remedied, e.g., by student mobility programs. Challenges related to physical presence did receive attention, and some solutions do have the potential of mitigating the current situation. In some cases, equipment can be scaled down to portable dimensions while preserving sufficient decision space and leaving students in control. In 2020, McHauser et al. [6] presented a Model-Factory-In-A$B o x$, a portable plant running a food industry production process. Cross-border shipping of goods has been less affected by the restrictions of 2020-2021, implying that travel limitations can indeed be remedied by portable equipment.

Location-bound physical equipment has also been examined earlier as a capacity bottleneck, leading to considerations of tailoring physical hands-on activities on site, and relocating other parts of the didactic program to virtual platforms or remote locations. Riemann et al. [7] compared physical and virtual learning factory counterparts, while Brinson [5] pointed out that in certain branches and skill types, remote or virtual didactic solutions may even perform better than traditional hands-on work. Examples for the use of virtual components have recently been presented by Nylund et al. [8] (an entirely virtual flexible manufacturing environment); Grube et al. [9] and Jara et al. [10] (digital twins with physical counterparts); Gong et al. [11] (extended reality, also in conjunction with physical hands-on activity); or García-Vela et al. [12] (comparable virtual vs. physical considerations in geosciences).

Another possibility of bridging travel restrictions is remote operation with connection to other facilities or participants. This has long been part of the Teaching Factory paradigm-in its factory-to-classroom track, remote presence is used for representing real-life production processes in the classroom [13]. Recently, a Teaching Factory Network has been proposed for exchange between multiple industrial and academic participants across several locations [14].

Blended learning, i.e., the variable combination of physical, virtual and remote learning has recently received much attention due to its relevance in coping with restricted personal attendance in the ongoing pandemic. Ożadowicz analyzed effects and potentials of blended learning in engineering higher education (automation in particular) [15], while Fleischmann [16] conducted investigations in education in the (i.e., design) domain. Notable differences have been found in the dynamics of students building up their own knowledge, depending on means and timing of theory and practical experience. Often, a flipped classroom appears more beneficial for distance learning — here, students first receive theoretical materials to be studied independently, while practical problems are then solved together, possibly via an online platform [15].

As it can be seen, past years have brought forth approaches that enable some of the site-bound learning factories to recover a part of their activities in times of attendance limitations. Developing and retaining such alternatives in postrestriction times will likely contribute to education opportunities with lasting enhancements [15, 1].Keeping to this assumption, the paper presents means of recovering parts of an earlier didactic offering by the example of a summer school program originally planned for 2020 [17]. While the entire two-week course is being prepared in collaboration of SZTAKI and Fraunhofer Austria, the paper is limited to measures taken at the sites of SZTAKI, with a focus on layout and process planning for human-robot collaborative assembly. The paper examines three dimensions in which mitigation measures can be taken: (1) tailoring of task decision space and complexity, (2) transition to virtual or offsite operation, and (3) organization and presence of students and education staff. While the examples presented are closely connected to a specific course, the same principles can be generalized for re-use in other comparable learning factory settings. The online version of the summer school program is planned to be rolled out in July/August 2021, therefore, the paper is of an in-progress nature, with feedback to be obtained upon completion of the course.

\section{Original course plan and impact of restrictions}

As originally planned, the part of the summer school on SZTAKI's premises foresees the elaboration of a solution for collaborative assembly of ball valves [17], by student teams of 3-4 persons each. The infrastructure comprises several workstations, each equipped with a collaborative robot, and a set of pallets and assembly fixtures. Students first 
become acquainted with theoretical background, the work environment, the task, and the equipment and technologies available. Next, teams design their own layout (arrangement of pallets and fixtures) and make their own choice of resource allocation (human or robot) to steps of the assembly process. Finally, measured runs of the assembly processes take place, and findings are analyzed, leading up to a comparative evaluation of all outcomes, with conclusions drawn together. A last time slot is reserved for experiments based on common conclusions.

The attendance and travel restrictions of the pandemic hampered the first roll-out of the course as originally planned. Nevertheless, possibilities were examined to recover as much as possible of the original activities by online/off-site alternatives. Much of the original plan depended strongly on (1) physical hands-on activities of students, and (2) consistent on-site presence of staff for support and supervision. In order to make the program transferable to remote locations, means of circumventing or reducing these constraints must be found. The next section examines dimensions where various levels of changes allow the program to be adapted to varying restrictions.

\section{Alternatives for online/remote courses}

\subsection{Tailoring complexity}

The original layout and process planning scenario is marked by implicit complexity and diversity of outcomes, requiring availability of co-located staff while students work with physical equipment. Possible layout and process choices are manifold, with potential unforeseen problems that are typically easily overcome with the help of experienced personnel. It is, however, circumstantial, if possible at all, to sufficiently replace this type of expertise-based help by a site-independent knowledge base or remote assistance. Instead, it is more viable to reduce complexity to a manageable degree, still leaving enough of a decision space to give the students the experience of "being in control".

A possible way of tailoring complexity is the discretization of choices. Most of this can be achieved in layout planning. Instead of a freely designed working area, standardized pallets are used at a set of pre-defined locations. The pallets themselves allow pre-defined fixtures — still with alternatives for the same assembly operation - to be placed in raster positions, and reoriented by multiples of $90^{\circ}$ (see Figure 1). This makes it easy to pre-generate robot motion and end effector pose/grip primitives that can undergo a feasibility check prior to the course. Similarly, process plans can rely on patterns which are elaborated beforehand, and keep students from steering into situations where co-location and live consultation with experienced personnel would be essential in tackling the problem. Simultaneously, the building-block approach still leaves room for the students' own decisions regarding both layout and process planning.

\subsection{Tools and techniques supporting virtual and remote learning}

Hands-on experience with physical equipment is not only an attractive facet of a learning factory course, but is also a crucial means of building up knowledge by the students' own initiative in discovery, experience and reflection. Therefore, it is important to recover as much as possible from (1) the "look-and-feel" of physical hands-on experience, and (2) the possibility of "being in control" and exploring cause-effect relations by one's own actions. To this end, distance-learning alternatives must be found that can convey necessary insight, and leave enough freedom for making decisions and seeing consequences. For the layout and process planning course in this example, the following tools and techniques have been identified:

Partial physical replication of the working environment-Many components of the working environment are FDMprinted in their original form, and the pallets are also easy to reproduce, given their simple geometry. Therefore, a physical replica of an important part of the entire workstation-i.e., the surfaces where assembly takes place-can be made available to remotely attending students at a reasonable cost. In a more optimistic case, students can receive a course preparation package by mail, containing a pallet, a set of fixtures, a functional gripper replica, and a workpiece kit. Should postal deliveries be impaired, it is still possible to share the design files of the components, and print them locally, presuming such capacities are available. 

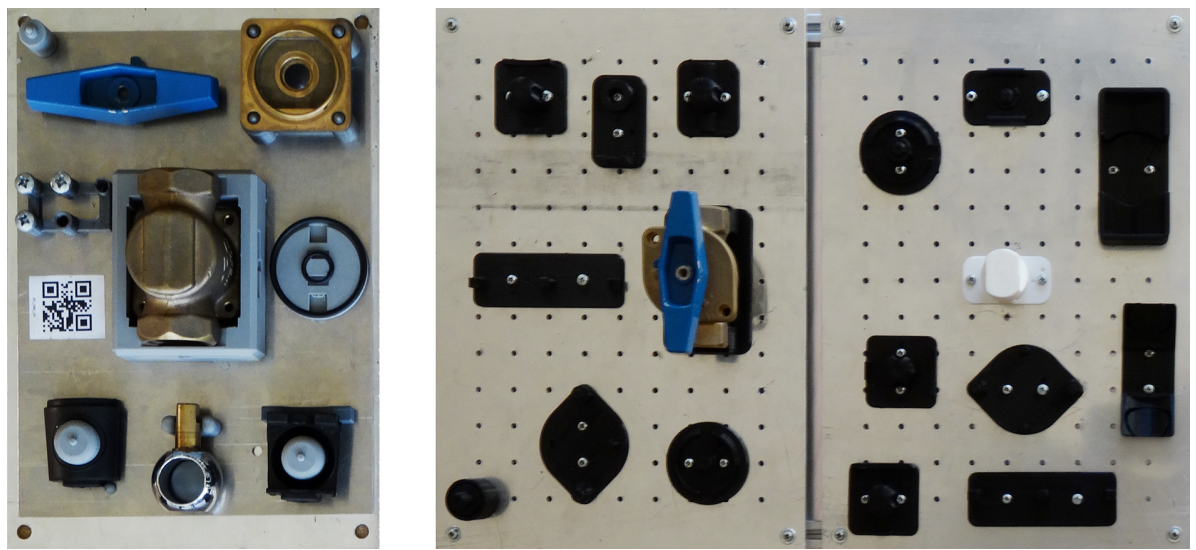

Fig. 1. Original arrangement of assembly pallet (left) and two different fixture layouts on rasterized pallets (right).

Virtual representations and tools-As described in prior publications [17], the scenario of the learning factory stems from a one-shot project, followed by other related R\&D activities focusing on the same application case. Therefore, a rich repository of virtual representations has already been elaborated, allowing a digital twin of the assembly station to be generated and made available to the participants of the course at reasonable effort. Experience with transition to simulation can also be gleaned from other tracks of didactic offering at the institute. Partly preceding the modification of the collaborative assembly course, the content of a single process scheduling lab exercise for another facility of SZTAKI (the Smart Factory [18]) has received a discrete event simulation and visualization tool, with the aim of integration into conventional curricula, in response to attendance restrictions at universities. The outcomes of other R\&D projects also contribute with tools allowing (1) calibration of the virtual model to a real scene via sensory input [19], (2) operation sequencing and path planning to assist in turning discrete layout and process choices into robot code [20,21,22], and (3) assessment of solutions with respect to collision constraints and performance criteria [22]. The problems these tools were originally developed for are far too complex and require too much prior experience and domain insight for a short course, but either their core algorithms, or their implemented functionalities can be adapted, and reproduced if needed, for the trimmed-down choices of the summer school application scenario. Much of these components are in-house developments without legal restrictions of use-commercial platforms will require license and access management that tailors admittance and permitted usage to the span of the course.

Adaptation of remote communication - During the 2020 lockdown, most universities have elaborated their standard ways of communicating with students in distance learning. Due to the prevailing character of today's higher education, this would rarely go beyond remotely held lectures, and simple communication and content sharing tools for project work. These platforms, however, are less suitable for real-time teamwork and consultation centered around locationbound physical equipment. As proposed above, a part of the hands-on work can be shifted to interaction with a virtual model, however, this is not always sufficient for teamwork normally targeting a physical system. This can be-at least partly-replaced with a blended mode of communication where personnel is co-located with the physical equipment, and participating students are connected via (1) live video granting sight of both on-site staff, and the physical plant, and (2) real-time coupling of the (calibrated) digital twin with the physical process. In this scenario, layout settings and robot programs elaborated remotely by the students are transferred to the physical equipment, and responses of the latter are mirrored by the digital twin during process execution. In a more enhanced case, comments or suggestions of instructors can also be visualized with the help of the digital twin, providing a remote substitute of on-site assistance.

\subsection{Reorganizing students and staff}

During 2020, countries throughout the world have experienced different levels of attendance and travel restrictions with varying impact on the possibilities available to an online course. In the most extreme-but very rare-case, lockdown measures do not even allow staff to be present in the physical facility. This would require a fully virtual 
course, and while some parts of the original course plan could still be recovered, the complete exclusion of the physical site would seriously challenge the original purpose of the program. In most countries, restrictions have peaked at all higher education being forced into distance-learning mode, while staff was still allowed on premises with distancing and protection measures. This situation would already allow remote connection of individual students with on-site personnel. Students would be sent individual course packages, and teamwork would be possible relying on the virtual tools presented in Subsection 3.2. In a more permissive case, only international or interregional travel restrictions would apply, allowing co-located teamwork of student groups in their respective locations. This would have the advantage of student teams elaborating their solutions in a more direct way, with more hands-on experience and easier communication. Given the substantial variation of lockdown measures, even between different countries at the same time, it is realistic to expect that the possibilities of students will vary by country. Therefore, flexibility must be allowed in formation and collaboration of different student teams.

\subsection{Course configuration for summer 2021}

A first run of the layout and process planning summer school program is planned to take place in late July and early August 2021. At the time of writing this paper, preparations of infrastructure, didactic content and background material are underway, and preliminary decisions regarding course configurations have been made. Given the one-year history of lockdown levels in Hungary, it is likely that staff will be allowed on site at the time of the course, and postal services across Europe will allow timely delivery of equipment to participants. Materials are being elaborated and planned to be distributed to support participation of student teams both with individual remote connection, and as a co-located local group, preparing to bridge differences in lockdown levels in participating countries.

Regardless of whichever case of restrictions will apply during the course, the timing and interdependence of elements in the summer school program have to be revised and adapted to the altered opportunities of communication, teamwork and hands-on experiments. Earlier work has already stressed the advantages of a flipped classroom arrangement, wherein students are acquainted with theoretical knowledge in independent work, and solve tasks afterwards in hands-on teamwork. This pattern is partly reflected in the modified order of activities suggested for the layout and process planning course. In a preparatory phase prior to the program, students already have access to didactic material, and hopefully have received their own assembly kits, so that first impressions can be gained in advance. The program linked to SZTAKI's premises begins with traditional lectures and virtual lab tours, whereafter students continue their acquaintance with background knowledge and can develop working hypotheses regarding solution elements on their own. This phase is followed by interactive teamwork when the solutions are built up both in the virtual models, and at the physical workstations with the help of local staff who also assist student teams in taking their solution to a working, measurable stage. Test runs and measurements are carried out in similar teamwork, whereafter findings are elaborated independently by student teams. A conclusive comparison of results and final corrective experiments-in response to problems surfaced during test runs or evaluation-are, again, carried out in the physical facility with the assistance of local personnel.

According to current plans, students will be allowed to keep their replicas and use them for their own projects, motivating them to give feedback to the organizers about further developments inspired by the summer school program.

\section{Conclusion and outlook}

Based on a specific example of a facility with collaborative assembly stations, the paper examined the feasibility and possible measures of moving the didactic offering of physical learning factories to distance learning arrangements, in an attempt to recover as much as possible of the hands-on character of learning factories. Such transformations were, and still are, necessary due to limitations of personal attendance during lockdowns in the COVID-19 pandemic. With the restrictions expected to continue throughout 2021, this is expected to be a lasting and relevant challenge for many learning factories. The transformation measures in the paper are gathered and assessed in the context of a specific layout and process planning summer school program, but characteristics of the students' tasks, the focal process and the equipment involved, suggest that the contribution of the paper-a step-wise catalog of measures for transition to 
virtual/remote education - can be adopted by a broader class of learning factories as well. At the time of writing the publication, the virtual summer school program is still undergoing detailed elaboration, hence, it is to be interpreted in an in-progress context, to be validated once results of the first run of the course are available.

\section{Acknowledgements}

Work for this paper was supported by the European Commission through the H2020 project EPIC under grant No.739592, and partly by two Hungarian national grants (1) iNEXT, under grant ED_18-22018-0006 titled "Research on prime exploitation of the potential provided by the industrial digitalisation", and (2) Thematic Excellence Programme 2020, TUDFO/51757/2019-ITM

\section{References}

[1] G. Marinoni, H. Vant Land, T. Jensen, The impact of Covid-19 on higher education around the world, IAU Global Survey Report.

[2] E. Abele, J. Metternich, M. Tisch, G. Chryssolouris, W. Sihn, H. ElMaraghy, V. Hummel, F. Ranz, Learning factories for research, education, and training, Procedia CIRP, 32 (2015) 1-6.

[3] J. Enke, M. Tisch, J. Metternich, Learning factory requirements analysis—Requirements of learning factory stakeholders on learning factories, Procedia CIRP, 55 (2016) 224-229.

[4] J. S. Lamancusa, T. W. Simpson, The learning factory-10 years of impact at penn state, in International Conference on Engineering Education, $16-21$.

[5] J. R. Brinson, Learning outcome achievement in non-traditional (virtual and remote) versus traditional (hands-on) laboratories: A review of the empirical research, Computers \& Education, 87 (2015) 218-237.

[6] L. McHauser, C. Schmitz, M. Hammer, Model-Factory-In-A-Box: A portable solution that brings the complexity of a real factory and all the benefits of experiential-learning environments directly to learners in industry, Procedia manufacturing, 45 (2020) $246-252$.

[7] T. Riemann, A. Kreß, L. Roth, S. Klipfel, J. Metternich, P. Grell, Agile Implementation of Virtual Reality in Learning Factories, Procedia Manufacturing, 45 (2020) 1-6.

[8] H. Nylund, V. Valjus, V. Toivonen, M. Lanz, H. Nieminen, The virtual FMS—an engineering education environment, Procedia manufacturing, 31 (2019) 251-257.

[9] D. Grube, A. A. Malik, A. Bilberg, SMEs can touch Industry 4.0 in the smart learning factory, Procedia Manufacturing, 31 (2019) $219-224$

[10] C. A. Jara, F. A. Candelas, S. T. Puente, F. Torres, Hands-on experiences of undergraduate students in Automatics and Robotics using a virtual and remote laboratory, Computers \& Education, 57(4) (2011) 2451-2461.

[11] L. Gong, Å. Fast-Berglund, B. Johansson, A Framework for Extended Reality System Development in Manufacturing, IEEE Access, 9 (2021) 24796-24813.

[12] M. García-Vela, J. Zambrano, D. Falquez, W. Pincay-Musso, K. Duque, N. Zumba, M. Barcia, J. Méndez, P. Valverde, P. Romero-Crespo, et al., Management Of Virtual Laboratory Experiments In The Geosciences Field In The Time Of COVID-19 Pandemic, in Proceedings of ICERI2020 Conference, vol. 9, 8702-8711.

[13] D. Mavrikios, K. Georgoulias, G. Chryssolouris, The teaching factory paradigm: Developments and outlook, Procedia Manufacturing, 23 (2018) 1-6.

[14] D. Mavrikios, K. Georgoulias, G. Chryssolouris, The Teaching Factory Network: A new collaborative paradigm for manufacturing education, Procedia Manufacturing, 31 (2019) 398-403.

[15] A. Ożadowicz, Modified Blended Learning in Engineering Higher Education during the COVID-19 LockdownBuilding Automation Courses Case Study, Education Sciences, 10(10) (2020) 292.

[16] K. Fleischmann, Hands-on versus virtual: Reshaping the design classroom with blended learning, Arts and Humanities in Higher Education, 2 (2020) 87-112.

[17] Zs. Kemény, R. Beregi, B. Tipary, K. Abai, J. Nacsa, Recent advances in learning content and infrastructure development for layout and process planning courses at the SZTAKI learning factories, Procedia Manufacturing, 45 (2020) 319-324.

[18] Zs. Kemény, R. J. Beregi, G. Erdős, J. Nacsa, The MTA SZTAKI Smart Factory: Platform for research and project-oriented skill development in higher education, Procedia CIRP, 54 (2016) 53-58.

[19] G. Erdős, I. Paniti, B. Tipary, Transformation of robotic workcells to digital twins, CIRP Annals, 69(1) (2020) $149-152$.

[20] G. Erdős, Cs. Kardos, Zs. Kemény, A. Kovács, J. Váncza, Process planning and offline programming for robotic remote laser welding systems, International Journal of Computer Integrated Manufacturing, 29(12) (2016) 1287-1306.

[21] D. Ceglarek, M. Colledani, J. Váncza, D.-Y. Kim, C. Marine, M. Kogel-Hollacher, A. Mistry, L. Bolognese, Rapid deployment of remote laser welding processes in automotive assembly systems, CIRP Annals, 64(1) (2015) 389-394.

[22] A. Kovács, Integrated task sequencing and path planning for robotic remote laser welding, International Journal of Production Research, 54(4) (2016) 1210-1224. 